\title{
Biomarkers in diabetic kidney disease: good use and piffalls
}

Silvia Titan'

1 Divisão de Nefrologia, Departamento de Clínica Médica, Faculdade de Medicina da Universidade de São Paulo (FMUSP), São Paulo, SP, Brasil

Correspondence to: SilviaTitan

smotitan@gmail.com

Received on Mar/28/2016 Accepted on Mar/28/2016

DOI: 10.1590/2359-3997000000167
$\mathrm{I}$ $\mathrm{n}$ this issue of Archives of Endocrinology and Metabolism (AE\&M), two manuscripts address the important issue of the search for new and reliable biomarkers of kidney function and chronic kidney disease $(\mathrm{CKD})$ risk in diabetes. Domingueti and cols. (1) evaluate the performance of several formulas of GFR estimation in regard to albuminuria classification (normo-, micro- and macroalbuminuria). In their analyses, cystatin C-based formulas showed the best performance, followed closely by the new CKD-EPI creatinine-based equation. Their findings highlight the crave for more precise measures of GFR estimation and waiving of less efficient methods such as Cockcroft-Gault formula. When compared to MDRD equation the new CKD-EPI equation is more accurate and reduces the known effect of MDRD on sub-estimating GFR in normal and near-normal values (2). However, the CKD-EPI equation based on creatinine also requires a race definition, which is always problematic, particularly in highly admixture populations. This formula has not been largely validated outside US, Europe and Australia and this should be done in populations ethnically different from the population in whom the equation was derived and revalidated, such as Brazil. Small Brazilian studies yielded results suggesting that this equation may be applied in our population (3-5), but larger studies are still necessary. On the other hand, cystatin $\mathrm{C}$ seems to improve accuracy in comparison to creatinine, but is still not largely available and its cost is still high. These problems could be minimized with a broader use of this marker, but to the present cystatin $\mathrm{C}$ use is still very much restricted to the research field.

In the other manuscript, Polat and cols. (6) have analyzed several endothelial dysfunction biomarkers in 73 patients with diabetes and either normoalbuminuria and microalbuminuria in comparison to healthy controls. The authors showed that although differences can be detected among the diabetic and non-diabetic groups, most biomarkers cannot discriminate those with and without albuminuria. Only sVCAM-1 showed a better performance in identifying those with microalbuminuria.

Reflections raised by the two papers come in accordance with a very actual discussion on diabetic kidney disease. One of the most fearest complications of long-term poor glycemic control, CKD in diabetes still faces old problems regarding two very basic problems: diagnosis and classification. Diagnosis has always been supported by the recognition of long term hyperglycemia, increasing albuminuria occurring along to GFR loss (represented by the hyperfiltration, microalbuminuric and macroalbuminuric phases), the presence of other microvascular injury, especially diabetic retinopathy, and absence of signs of other kidney diseases. However, it has been recently recognized that the classical description of the hyperfiltration, micro- and macroalbuminuric phases is limited, since a significant number of diabetic patients with CKD (and losing GFR over time) may in fact present normoalbuminuria and or micro- 
albuminuria throughout years and never progress to the macroalbuminuric phase (7). Normoalbuminuric and microalbuminuric patients which GFR reduction tend to present a slower decline of GFR and better glycemic control history than those who present macroalbuminuria (7), but even so represent a significant proportion of both type 1 and type 2 CKD patients (7-10). The concept of a progressive renal decline has also been contested $(11,12)$, at least in type 1 diabetes. In addition, recent trials have questioned the use of albuminuria as a surrogate marker of CKD by showing that GFR loss and albuminuria increase may be uncoupled in diabetic and non-diabetic CKD $(13,14)$. By replacing renal function assessment for albuminuria outcomes so extensively in the last 20 years we have generated some misleading results. Emphasis has been made in the need of using again "hard renal outcomes" (such as renal replacement therapy need and doubling of creatinine) instead of surrogate markers, particularly in clinical trials. It has been proposed that the term diabetic nephropathy should be replaced by the term diabetic kidney disease or diabetes-related kidney diseases, although societies throughout the world have not reached a consensus. This new term should include a more broad comprehension of renal injury occurring in diabetes, contemplating different clinical, laboratorial, pathological and prognostic conditions. In the same way, the use of albuminuria as a continuous marker of risk seems to add more information than the simple and rigid classification of albuminuria in normo-, microand macroalbuminuria (15).

By using albuminuria as the main outcome measure, the two papers in this issue of AE\&M stumble into this problematic. Ideally, performance of different ways of estimating GFR should be validated by a gold-standard, such as inulin clearance or radioisotope decay curves, exams which are extremely laborious and time-consuming. Performance should also be evaluated regarding prediction of renal and cardiovascular hard clinical events, requiring long term costly studies with large sample size. Therefore, surrogate markers are still needed and will continue being used, particularly in experimental and small clinical studies. Albuminuria has been extensively demonstrated to be an independent predictor of CKD progression in all etiologies of albuminuric kidney diseases and is clearly the best CKD surrogate marker we have so far. Data generated from studies using surrogate markers yield a great amount of information to our understanding of diabetes-re- lated complications. However, when it comes to the establishment of more definite therapeutic and epidemiological strategies, we have to consider the whole spectrum of diabetic kidney disease, and "hard clinical events" should be prioritized as outcomes.

The search for new biomarkers in CKD is intense in the literature. The performance of several tubular markers, urinary and serum inflammatory cytokines, endothelial and podocyte biomarkers, metabolites analyzed by metabolomics, and genetic polymorphism's panels are currently being evaluated for the prediction of CKD risk. More accurate identification of those at risk along with a better understanding of the pathophysiology of CKD progression are two fundamental steps for generating new therapeutic tools and for improving health care in CKD and in diabetic kidney disease.

Disclosure: Advisory Board para Novartis 2014.

\section{REFERENCES}

1. Domingueti $C P$, Fóscolo RB, Simões e Silva AC, Dusse LMS, Reis JS, Carvalho MG, et al. Evaluation of creatinine-based and cystatin C-based equations for estimation of glomerular filtration rate in type 1 diabetic patients. Arch Endocrinol Metab. 2016;60(2):108-16.

2. Levey AS, Inker LA, Coresh J. GFR estimation: from physiology to public health. Am J Kidney Dis. 2014;63(5):820-34.

3. Veronese FV, Gomes EC, Chanan J, Carraro MA, Camargo EG, Soares AA, et al. Performance of CKD-EPI equation to estimate glomerular filtration rate as compared to MDRD equation in South Brazilian individuals in each stage of renal function. Clin Chem Lab Med. 2014;52(12):1747-54.

4. Lopes MB, Araújo LQ, Passos MT, Nishida SK, Kirsztajn GM, Cendoroglo MS, et al. Estimation of glomerular filtration rate from serum creatinine and cystatin $\mathrm{C}$ in octogenarians and nonagenarians. BMC Nephrol. 2013;14:265.

5. Silveiro SP, Araújo GN, Ferreira MN, Souza FD, Yamaguchi HM, Camargo EG. Chronic Kidney Disease Epidemiology Collaboration (CKD-EPI) equation pronouncedly underestimates glomerular filtration rate in type 2 diabetes. Diabetes Care. 2011;34(11):2353-5.

6. Polat SB, Ugurlu N, Aslan N, Cuhaci N, Ersoy R, Cakir B.Evaluation of biochemical and clinical markers of endotelial dysfunction and their correlation with urinary albumin excretion in patients with type 1 diabetes mellitus. Arch Endocrinol Metab. 2016;60(2):117-24.

7. Perkins BA, Ficociello LH, Roshan B, Warram JH, Krolewski AS. In patients with type 1 diabetes and new-onset microalbuminuria the development of advanced chronic kidney disease may not require progression to proteinuria. Kidney Int. 2010;77(1):57-64.

8. Kramer HJ, Nguyen QD, Curhan G, Hsu CY. Renal insufficiency in the absence of albuminuria and retinopathy among adults with type 2 diabetes mellitus. JAMA. 2003;289(24):3273-7.

9. Thomas MC, Macisaac RJ, Jerums G, Weekes A, Moran J, Shaw $\mathrm{JE}$, et al. Nonalbuminuric renal impairment in type 2 diabetic patients and in the general population (national evaluation of the frequency of renal impairment cO-existing with NIDDM [NEFRON] 11). Diabetes Care. 2009;32(8):1497-502. 
10. Rigalleau V, Lasseur C, Raffaitin C, Beauvieux MC, Barthe N, Chauveau $P$, et al. Normoalbuminuric renal-insufficient diabetic patients: a lower-risk group. Diabetes Care. 2007;30(8):2034-9.

11. Krolewski AS. Progressive renal decline: the new paradigm of diabetic nephropathy in type 1 diabetes. Diabetes Care. 2015;38(6):954-62.

12. Perkins BA, Ficociello LH, Ostrander BE, Silva KH, Weinberg J, Warram $\mathrm{JH}$, et al. Microalbuminuria and the risk for early progressive renal function decline in type 1 diabetes. J Am Soc Nephrol. 2007;18(4):1353-61.
13. Mann JF, Schmieder RE, McQueen M, Dyal L, Schumacher H, Pogue J, et al.; ONTARGET investigators. Renal outcomes with telmisartan, ramipril, or both, in people at high vascular risk (the ONTARGET study): a multicentre, randomised, double-blind, controlled trial. Lancet. 2008;372(9638):547-53.

14. Mann JF, Schmieder RE, Dyal L, McQueen MJ, Schumacher $H$, Pogue J, et al.; TRANSCEND (Telmisartan Randomised Assessment Study in ACE Intolerant Subjects with Cardiovascular Disease) Investigators. Effect of telmisartan on renal outcomes: a randomized trial. Ann Intern Med. 2009;151(1):1-10, W1-2.

15. Ruggenenti $P$, Remuzzi G. Time to abandon microalbuminuria? Kidney Int. 2006;70(7):1214-22. 